\title{
EVALUACIÓN POSTOPERATORIA TRAS LA APLICACIÓN INTRAALVEOLAR DE PLASMA RICO EN FACTORES DE CRECIMIENTO POSTEXTRACCIÓN DE TERCEROS MOLARES EN LA UNIDAD ACADÉMICA DE CIENCIA ODONTOLÓGICA, 2014-2015
}

SARMIENTO-CÁRDENAS DAVID *

Recibido: 06/09/2015

PIEDRA-SARMIENTO XAVIER **

Aceptado: 13/10/2015

\begin{abstract}
RESUMEN
Objetivo: Determinar la eficacia y beneficios de la colocación intraalveolar del Plasma Rico en Plaquetas con Factores de Crecimiento en Cirugía de Tercer Molar Inferior (PRPFC), en base a los signos y síntomas postoperatorios inmediatos. Materiales y Métodos: Es de corte transversal de tipo descriptivo, experimental con ciego simple. El universo fue de 280 pacientes, cuya muestra fue de 26, con 52 terceros molares inferiores respectivamente. Resultados: Al realizar el control post operatorio de los dos terceros molares inferiores y al usar PRPFC, al tercer día se evidencia un edema de leve a moderado; $y$ al no ser aplicado el PRPFC $(p<0.001)$ se observa un edema severo. Hubo hallazgos clínicos satisfactorios en relación a la cicatrización de los tejidos blandos tras su aplicación. Relacionando el dolor con el uso de PRPFC en cirugía de tercer molar no existe significancia estadística por la subjetividad de la variable $(p<0.308)$. Conclusiones: El uso de PRPFC en cirugía de tercer molar inferior es una terapia viable, sin riesgos y asequible; obteniéndose resultados positivos favoreciendo el proceso de cicatrización de tejidos blandos, conllevando a una recuperación postoperatoria menos traumática.
\end{abstract}

Palabras clave: plasma, plaquetas, factores de crecimiento, tercer molar, edema e inflamación.

\section{ABSTRACT}

This study aims to determine the effectiveness and benefits of the intra-alveolar placement of Platelet Plasma Rich in Growth Factors (PRPGF) on third lower molar surgery, based on the immediate postoperative signs and symptoms. It is a cross-sectional, descriptive, experimental, and single-blind study. The universe comprised 280 patients and the sample was 26 with 52 lower third molars. Results: The postoperative control of the two third molars using PRPGF on the third day showed a mild to moderate edema; when PRPGF was not applied $(p<0.001)$ severe edema was observed $(p<0.001)$. There were satisfactory clinical findings regarding healing of soft tissue after application. Pain relating to the use of PRPFC in third molar surgery there is no statistical significance by the subjectivity of the variable $(p=0.308)$. Conclusions: The use of PRPFC in lower third molar surgery is a viable therapy, safe and affordable; yielding positive results favoring the healing process of soft tissues, leading to a less traumatic postoperative recovery.

Keywords: plasma, platelets, growth factors, third molar, edema and inflammation.

\section{INTRODUCCIÓN}

Los procedimientos quirúrgicos dentro de la Odontología implican altas tasas de morbilidad, varios autores durante mucho tiempo han buscado diversas técnicas y terapias coadyuvantes para dichos procedimientos, el hecho de buscar una terapia autóloga despierta el interés de varios operadores, con diversos conceptos aplicados a la odontología. El concepto del Plasma Rico en Plaquetas con Factores de Crecimiento (PRPFC) se viene desarrollando desde hace varios años, Cohen y Levi-Montalcini en la década del 50, descubren los factores de crecimiento (FC) nervioso, que les otorgó el premio nobel en 1986, antes en 1962 el mismo Cohen propone el concepto de los factores de crecimiento epidérmico, estos hechos se consideran un hito básico para el desarrollo del estudio de los factores de crecimiento como tal y de la reparación ante una herida. ${ }^{1-4}$ Así surge el estudio de los factores de crecimiento plaquetario mediante estudios claves por Raines y Ross, de BowenPope y Ross quienes detallaron analíticamente tanto la definición como la capacidad de unión a células cultivadas, Tayapongsak en 1994 centra su atención sobre los mecanismos intrínsecos de la respuesta celular, pero es Marx que en 1998 estudia a las plaquetas y a los FC como los responsables de la reparación celular, en ese momento son descubiertos los factores de crecimiento plaquetario que hoy conocemos, es Anitua que en 1999 propone usar al PRPFC por sus capacidades regenerativas en varias áreas médicas y de interés, entre una de ellas la cirugía oral y maxilofacial. ${ }^{4-6}$

\footnotetext{
* Odontólogo egresado Odontología, Universidad Católica de Cuenca.Ecuador

** Especialista en Cirugía Oral, Catedrática de la Universidad Católica de Cuenca.Ecuador
} 
Al PRPFC se le considera un concentrado plaquetario, entendiéndose como un producto autólogo, no homólogo ${ }^{1}$ es una concentración plaquetaria en un pequeño volumen de plasma, con una cantidad de 2 a 5 veces más plaquetas que en un estado basal, que favorece a diversos procesos neoformativos y conductivos dirigidos por los factores de crecimiento de origen plaquetario, siendo un concentrado de células con propiedades moduladoras y estimuladoras de la proliferación de células madre de origen mesenquimal como son los fibroblastos, osteoblastos, células endoteliales, células epiteliales, adipoblastos, miocitos y condrocitos. ${ }^{1-13}$ Los Factores de crecimiento, se encuentran dentro de los gránulos alfa plaquetarios, que al ser estimulados son liberados dichos factores de crecimiento al medio externo mediante exocitosis para iniciar todo un proceso de regeneración gracias a la variedad de factores de crecimiento, como es el factor de crecimiento de origen plaquetario, el factor de crecimiento de transformación-beta, el factor de crecimiento fibroblástico, el factor de crecimiento similar a la insulina, el factor de crecimiento endotelial vascular y el factor de crecimiento epidérmico. ${ }^{2-4,7,10-12,14-15}$

La acción de los factores de crecimiento dependen del tipo de células y el grado de madurez ${ }^{3}$ el factor de crecimiento beta 1 promueve la síntesis de la matriz extracelular e induce la expresión de receptores para el factor de crecimiento plaquetario, que en conjunto estimulan la síntesis de colágeno tipo 1, fibronectina y osteonectina y a la vez la quimiotaxis, también disminuyen la síntesis de métalo-proteínas que son quienes degradan la matriz extracelular, todos estos hechos en conjunto tienen la acción de disminuir la destrucción de la matriz de tejido conjuntivo, el factor de crecimiento de transformación beta 1 además inhibe la formación de osteoclastos pero por otro lado contribuye a la resorción del hueso por un mecanismo dependiente de las prostaglandinas, es un proceso complejo pero todo indica que es uno de los factores de conexión entre la reabsorción y formación ósea. ${ }^{4}$

La presencia del factores de crecimiento epidérmico es casi indetectable pero estos participan en las primeras fases de reparación, estimulando la migración y división celular y aumentando la síntesis de proteínas como la fibronectina ${ }^{4}$ por otro lado el factores de crecimiento similar a la insulina participa como un potente agente quimio-táctico para las células vasculares endoteliales, provocando la neovascularización de la herida, así también en la proliferación y diferenciación de osteoblastos, el factor de crecimiento endotelial vascular estimula la proliferación celular en aquellos vasos sanguíneos que han sido dañados. 2-4, 7, 10-12, 14, 16-18

Protocolo de Anitua: Se trata de un procedimiento con una sola centrifugación a 1800 revoluciones por minuto y durante $8 \mathrm{~min}$, se utilizan tubos estériles con citrato de sodio al $8 \%$ agente anticoagulante que mediante la fuerza centrífuga permitirá la separación de los elementos sanguíneos, posteriormente se realiza el pipeteo con pipeta automática de 500 microlitros (ul), para las dos primeras fracciones y la tercera fracción a ser utilizada se realizan pipeteos de 100 microlitros, tomando solamente el producto plasmático, Anitua recomienda no utilizar la serie blanca, por la presencia de células proinflamatorias y tampoco usar la serie roja ya que producirá hemólisis del coágulo obtenido además de hacerlo inestable, se lo lleva a un tubo estéril en donde se procede a la activación plaquetaria exógena con cloruro cálcico o gluconato de calcio los dos al 10\% en cantidades de 0.05 microlitros por cada mililitro de PRP obtenido, para lo cual se utilizan micropipetas automáticas, estos agentes cálcicos provocan la activación y degranulación plaquetaria procedimiento que fue antes mencionado y por ende la activación de todos los factores de crecimiento, formando un gel plaquetario, el cual expulsa de su interior los factores de crecimiento en los primeros 10 a 15 minutos casi en su totalidad y durante la primera hora tiene un uso y aplicación favorable, posterior al tiempo planteado dejará de ser útil fuera del lecho quirúrgico. 2-3, 5-7, 10-12

Obsérvese la imagen 1 de la colocación intraalveolar de PRPFC dentro del lecho quirúrgico en la presente investigación. Las plaquetas comienzan a secretar activamente los FC en los 10 primeros minutos, liberando más del $95 \%$ de los factores de crecimiento pre sintetizados en el lapso de 1 hora. Tras esta liberación proteica en el lapso de una hora las plaquetas han sintetizado la mayoría de proteínas y adicionalmente secretan la misma durante un lapso de 5 a 10 días más, ya en el lecho de acción, el PRPFC actúa además como una quimio táctica para los macrófagos ${ }^{4,7}$.

Momentos de regeneración: Los mecanismos fundamentales en la liberación de los factores decrecimiento en concentrados plaquetarios se dan por difusión, la concentración temporal y una distribución espacial exacta varía en función de la infiltración de los tejidos durante la respuesta inicial de reparación, teniendo en cuenta a dichos factores como claves para el éxito, es así que la activación y presencia puntual en el momento y concentración exacto ante el inicio de la cascada de regeneración va de la mano con resultados satisfactorios ${ }^{2,4}$. La colocación se realiza entre los 10 y 15 minutos posteriores a la activación plaquetaria, colocando en el lecho quirúrgico un coágulo de PRPFC que ha sido entramado por una malla de fibrina interna que nos permite manipular mientras dure esta unión, al ser colocado el mismo, favorece a un cuadro de hipoxia respecto al lecho receptor que estuvo bien oxigenado, la consecuente disminución del pH de 4 o 6 respecto al lecho receptor cuyo $\mathrm{pH}$ es de 7 , momento en el cual inicia la revascularización de la zona de migración de 
células pluri-potenciales, células ósteo-componentes y la mito-génesis de células ósteo-progenitoras y fibroblastos 2,4

\section{MATERIALES Y MÉTODOS}

Previa inclusión de los pacientes al estudio se tomó en cuenta que cumplan con: estado de salud normal o sano, biometría hemática actualizada, pacientes con extracción indicada de los dos terceros molares inferiores, edad de 18 a 24 años.

Obtención del PRPFC: Se usó el protocolo de Anitua, procediendo a la obtención de plasma rico en plaquetas con factores de crecimiento y la colocación intraalveolar post extracción de terceros molares inferiores, se utilizó una centrifugadora K-GEMY III, tubos vacutainer tapa celeste, tubos estériles tapa roja para la preparación del PRPFC, pipetas automáticas marca ACUMAX PRO de 500, 100 y $50 \mathrm{ul}$, ampollas de gluconato de calcio al $10 \%$ para cada paciente.

\section{Terapia farmacológica}

- Meloxicam en ampolla de 15 mg. Vía intramuscular. 1 ampolla única después de la intervención quirúrgica.

- Amoxicilina/Ácido Clavulánico de 1 gr. cada 8 horas durante 7 días. Vía oral.

- Ibuprofeno de $600 \mathrm{mg}$. cada 8 horas por 4 días. Vía oral.

Valoración postoperatoria a cada paciente: La evaluación clínica de los signos y síntomas pre y posoperatorios se la realizó mediante, revisiones clínicas, fotografías intraorales y extraorales con una cámara de fotos semiprofesional Samsung Smart Camera, medición con cinta, escala del dolor, escala visual análoga (EVA).

La medición del grado de inflamación y edema: El grado de edema fue medido en relación a puntos anatómicos. Basándonos en mediciones utilizadas por Iria ${ }^{4}$ en un estudio piloto realizado en la Universidad Complutense de Madrid en la Facultad de Odontología, Departamento de Medicina Bucal, realizando cierta variación en los puntos anatómicos.

La cantidad de edema fue valorado en relación a una medición tanto pre y postoperatoria de cada lado, mediante la medición extra oral desde los puntos anatómicos que van de tragus- gonion -mentón (línea media) que fueron medidos en un momento preoperatorio y al 3er día posoperatorio considerado el día de mayor inflamación, manteniendo la siguiente escala de medición:

- $\quad$ Edema leve: Medida postquirúrgica es $\leq$ a $0.5 \mathrm{~cm}$ en relación a medida pre quirúrgica.

- Edema Moderado: Medida postquirúrgica aumenta de 0.5 a $1.5 \mathrm{~cm}$. mayor en relación a medida pre quirúrgica.

- Edema Severo: Medida postquirúrgica aumenta más de $1.5 \mathrm{~cm}$. en relación a medida pre quirúrgica.

Al séptimo día pos operatorio se realizó el retiro de los puntos de sutura y al octavo día la toma de fotografías intraorales para la valoración de los procesos de cicatrización, ausencia o presencia de fluidos sanguinolentos a la palpación y el estado o la proximidad de los extremos de la herida, además se sondeó al paciente sobre el proceso de recuperación. La medición del dolor: Se realizó la Escala Visual Análoga que consta un ciclo lineal del cero al diez, el valor 0 significa ausencia total de dolor, de 1 a 3 dolor leve, de 4 a 7 dolor moderado, de 8 a 10 dolor severo.

\section{RESULTADOS}

En este estudio participaron 26 personas, 16 de sexo femenino y 10 de sexo masculino, con un promedio de edad entre $19.94 \pm 1.5$ años y $20.80 \pm 2.3$; teniendo una edad mínima de 18 años y máxima de 24 años. No se encontraron diferencias estadísticamente significativas entre las edades de ambos géneros. $(p=0.362)$. Según la clasificación de Winter, en el grupo femenino el mayor porcentaje estuvo posicionado mesioangularmente $(62.50 \%)$, y en el sexo masculino el mayor porcentaje estuvo posicionado horizontalmente (50.00\%). Y el grupo que no recibió plasma rico en plaquetas con factores de crecimiento, tanto varones como mujeres, el mayor porcentaje estuvo posicionado mesioangularmente ( $81.25 \%$ y $50.00 \%$ respectivamente). (Tabla 1 ) 
TABLA 1. Posición de las piezas dentarias según la Clasificación de Winter de acuerdo al sexo.

\begin{tabular}{lcccccc}
\multicolumn{1}{c}{ Clasificación de Winter } & \multicolumn{2}{c}{ Femenino } & \multicolumn{2}{c}{ Masculino } & \multicolumn{2}{c}{ Total } \\
& $\mathbf{n}$ & $\%$ & $\mathbf{n}$ & $\%$ & $\mathbf{n}$ & $\%$ \\
\hline Si Plasma rico en Plaquetas & & & & & & \\
$\quad$ Vertical & 3 & 18.75 & 2 & 20.00 & 5 & 19.23 \\
$\quad$ Mesioangular & 10 & 62.50 & 3 & 30.00 & 13 & 50.00 \\
$\quad$ Horizontal & 3 & 18.75 & 5 & 50.00 & 8 & 30.77 \\
$\quad$ Total & 16 & 100.00 & 10 & 100.00 & 26 & 100.00 \\
No Plasma rico en Plaquetas & & & & & & \\
$\quad$ Vertical & 2 & 12.50 & 2 & 20.00 & 4 & 15.38 \\
$\quad$ Mesioangular & 13 & 81.25 & 5 & 50.00 & 18 & 69.23 \\
$\quad$ Horizontal & 1 & 6.25 & 3 & 30.00 & 4 & 15.38 \\
$\quad$ Total & 16 & 100.00 & 10 & 100.00 & 26 & 100.00 \\
\hline
\end{tabular}

Según la Clasificación de Pell y Gregory, tomando en cuenta el género, en el grupo que recibió plasma rico en plaquetas con factores de crecimiento, tenemos que, en el sexo femenino el mayor porcentaje estuvo posicionado en B II (56.25\%), y en el sexo masculino el mayor porcentaje estuvo posicionado en A I y B II (30\% respectivamente). En el grupo que no recibió Plasma rico en plaquetas con factores de crecimiento, en el sexo femenino y masculino, el mayor porcentaje estuvo posicionado en B II (75.00\% y $40.00 \%$ respectivamente). (Tabla 2).

TABLA 2. Posición de las piezas dentarias según la Clasificación de Pell y Gregory.

\begin{tabular}{|c|c|c|c|c|c|c|}
\hline \multirow{2}{*}{$\begin{array}{c}\text { Clasificación de Pell y } \\
\text { Gregory }\end{array}$} & \multirow{2}{*}{\multicolumn{2}{|c|}{ Femenino }} & \multirow{2}{*}{\multicolumn{2}{|c|}{ Masculino }} & \multirow{2}{*}{\multicolumn{2}{|c|}{ Total }} \\
\hline & & & & & & \\
\hline \multicolumn{7}{|l|}{ Si Plasma rico en Plaquetas } \\
\hline AII & 1 & 6.25 & 3 & 30.00 & 4 & 15.38 \\
\hline A III & 3 & 18.75 & 1 & 10.00 & 4 & 15.38 \\
\hline B I & 2 & 12.50 & 2 & 20.00 & 4 & 15.38 \\
\hline B ॥ & 9 & 56.25 & 3 & 30.00 & 12 & 46.15 \\
\hline B III & 1 & 6.25 & 1 & 10.00 & 2 & 7.69 \\
\hline Total & 16 & 100.00 & 10 & 100.00 & 26 & 100.00 \\
\hline \multicolumn{7}{|l|}{ No Plasma rico en Plaquetas } \\
\hline AII & 1 & 6.25 & 2 & 20.00 & 3 & 11.54 \\
\hline A III & 3 & 18.75 & 2 & 20.00 & 5 & 19.23 \\
\hline B I & 0 & 0.00 & 2 & 20.00 & 2 & 7.69 \\
\hline B II & 12 & 75.00 & 4 & 40.00 & 16 & 61.54 \\
\hline Total & 16 & 100.00 & 10 & 100.00 & 26 & 100.00 \\
\hline
\end{tabular}

TABLA 3. Nivel de edema en pacientes con y sin tratamiento PRPFC intraalveolar en cirugía de tercer molar inferior.

\begin{tabular}{|c|c|c|c|c|c|c|}
\hline \multirow{3}{*}{ Edema } & \multicolumn{4}{|c|}{ Plasma rico en Plaquetas } & \multirow{2}{*}{\multicolumn{2}{|c|}{ Total }} \\
\hline & \multicolumn{2}{|c|}{$\mathrm{Si}$} & \multicolumn{2}{|c|}{ No } & & \\
\hline & $\mathrm{n}$ & $\%$ & $\mathrm{n}$ & $\%$ & $\mathrm{n}$ & $\%$ \\
\hline Leve & $\overline{17}$ & 65.38 & 0 & 0.00 & $\overline{17}$ & 32.69 \\
\hline Moderado & 9 & 34.62 & 18 & 69.23 & 27 & 51.92 \\
\hline Severo & 0 & 0.00 & 8 & 30.77 & 8 & 15.38 \\
\hline Total & 26 & 100.00 & 26 & 100.00 & 52 & 100.00 \\
\hline
\end{tabular}


De los individuos que recibieron plasma rico en plaquetas con factores de crecimiento, el $26.92 \%$, presentó un nivel de dolor leve; el $61.54 \%$ dolor moderado y el $11.54 \%$ dolor severo. En los pacientes que no recibieron tratamiento el $15.38 \%$ presentó dolor leve, el $50 \%$ dolor moderado y el $34.62 \%$ dolor severo. No se encontró asociación estadísticamente significativa entre recibir plasma rico en plaquetas con factores de crecimiento y presencia de dolor postoperatorio después de una cirugía de tercer molar inferior. $(p=0.308)$. (Tabla 4).

TABLA 4. Relación entre el Nivel de Dolor de los pacientes con y sin tratamiento con PRPFC intraalveolar en cirugía de tercer molar inferior.

\begin{tabular}{ccccccc}
\hline \multirow{2}{*}{ Dolor } & \multicolumn{3}{c}{ Plasma rico en Plaquetas } & \multicolumn{2}{c}{ Total } \\
& $\mathrm{n}$ & $\mathrm{Si}$ & $\mathrm{n}$ & $\%$ & $\mathrm{n}$ & $\%$ \\
\hline Leve & 7 & 26.92 & 4 & 15.38 & 11 & 21.15 \\
Moderado & 16 & 61.54 & 13 & 50.00 & 29 & 55.77 \\
Severo & 3 & 11.54 & 9 & 34.62 & 12 & 23.08 \\
Total & 26 & 100.00 & 26 & 100.00 & 52 & 100.00 \\
\hline
\end{tabular}

En la tabla 5 se evidencia una relación del grado de edema con la posición según Winter del tercer molar inferior y genero de los pacientes, se observa que el sexo femenino que usó PRPFC intraalveolar el mayor porcentaje está en la posición mesioangular con un $31.25 \%$ el mismo presentó un edema entre leve y moderado. Por el contrario, los pacientes de sexo femenino que no recibieron PRPFC presentan un mayor porcentaje en posición mesioangular, con un $50 \%$ de edema moderado y un $31.35 \%$ de severidad en el edema. De los pacientes de sexo masculino que usaron PRPFC intraalveolar el mayor porcentaje está en la posición mesioangular con un $30 \%$ con edema leve, y un $30 \%$ con edema moderado en posición horizontal y cero severidad en el edema. Los pacientes masculinos que no recibieron PRPFC, presentan un mayor porcentaje en posición mesioangular, con un $50 \%$ de edema moderado y un $10 \%$ de edema severo en posición vertical y posición horizontal respectivamente.

TABLA 5. Relación entre el Nivel de Edema, género y clasificación de Winter de los pacientes con y sin tratamiento con PRPFC intraalveolar en cirugía de tercer molar inferior.

\begin{tabular}{|c|c|c|c|c|c|c|c|c|c|}
\hline \multirow[t]{2}{*}{ SEXO } & \multirow{2}{*}{ Clasificación de Winter } & \multicolumn{4}{|c|}{$\begin{array}{c}\text { EDEMA } \\
\text { Moderado }\end{array}$} & \multicolumn{2}{|c|}{ Severo } & \multicolumn{2}{|c|}{ Total } \\
\hline & & $\mathrm{n}$ & $\%$ & $\mathrm{n}$ & $\%$ & n & $\%$ & $\mathrm{n}$ & $\%$ \\
\hline \multicolumn{10}{|c|}{ Femenino } \\
\hline \multicolumn{10}{|c|}{ Si Plasma rico en Plaquetas } \\
\hline & Vertical & 2 & 12,50 & 1 & 6.25 & 0 & 0.00 & 3 & 18,75 \\
\hline & Mesioangular & 5 & 31,25 & 5 & 31,25 & 0 & 0,00 & 10 & 62,50 \\
\hline & Horizontal & 3 & 18,75 & 0 & 0,00 & 0 & 0,00 & 3 & 18,75 \\
\hline & Total & 10 & 62,50 & 6 & 37,50 & 0 & 0,00 & 16 & 100,00 \\
\hline \multicolumn{10}{|c|}{ No Plasma rico en Plaquetas } \\
\hline & Vertical & 0 & 0,00 & 2 & 12,50 & 0 & 0,00 & 2 & 12,50 \\
\hline & Mesioangular & 0 & 0,00 & 8 & 50,00 & 5 & 31,25 & 13 & 81,25 \\
\hline & Horizontal & 0 & 0,00 & 0 & 0,00 & 1 & 6.25 & 1 & 6.25 \\
\hline & Total & 0 & 0,00 & 10 & 62,50 & 6 & 37,50 & 16 & 100,00 \\
\hline
\end{tabular}

Masculino

Si Plasma rico en Plaauetas

\begin{tabular}{lcccccccc} 
Vertical & 2 & 20,00 & 0 & 0,00 & 0 & 0,00 & 2 & 20,00 \\
Mesioangular & 3 & 30,00 & 0 & 0,00 & 0 & 0,00 & 3 & 30,00 \\
Horizontal & 2 & 20,00 & 3 & 30,00 & 0 & 0,00 & 5 & 50,00 \\
$\quad \begin{array}{l}\text { Total } \\
\text { Plasma rico en Plaquetas }\end{array}$ & 7 & 70,00 & 3 & 30,00 & 0 & 0,00 & 10 & 100,00 \\
$\begin{array}{l}\text { Vertical } \\
\text { Mesioangular }\end{array}$ & 0 & 0,00 & 1 & 10,00 & 1 & 10,00 & 2 & 20,00 \\
Horizontal & 0 & 0,00 & 5 & 50,00 & 0 & 0,00 & 5 & 50,00 \\
Total & 0 & 0,00 & 2 & 20,00 & 1 & 10,00 & 3 & 30,00 \\
& 0 & 0,00 & 8 & 80,00 & 2 & 20,00 & 10 & 100,00 \\
\hline
\end{tabular}

En la tabla 6 se relaciona el nivel de severidad del edema con la posición (según Pell y Gregory) del tercer molar inferior, tomando en cuenta el sexo de los pacientes; nos indica que, los pacientes de sexo femenino que 
usaron PRPFC intraalveolar el mayor porcentaje está en la posición B II con un $25 \%$ que presento un edema leve, moderado con un $31.35 \%$ e inexistencia de severidad. Los pacientes de sexo femenino que no recibieron PRPFC, presentan un mayor porcentaje en posición B II, con un valor de $37.50 \%$ en ambos casos tanto moderado como severo. Entre los pacientes de sexo masculino que usaron PRPFC intraalveolar, el mayor porcentaje está en la posición A I - B I y B II con un 20\% presentando edema leve, y moderado un $10 \%$ en posición A I - B II y B III y no registrándose severidad en el edema. Los pacientes que no recibieron PRPFC de sexo masculino, presentan un mayor porcentaje en posición B II con un $30 \%$ de edema moderado y un $10 \%$ de edema severo en posición A II y B II.

TABLA 6. Relación entre el nivel de Edema, sexo y clasificación de Pell y Gregory de los pacientes con y sin tratamiento con PRPFC intraalveolar en cirugía de tercer molar inferior.

\begin{tabular}{|c|c|c|c|c|c|c|c|c|c|}
\hline \multirow{3}{*}{ SEXO } & \multirow{3}{*}{$\begin{array}{c}\text { Clasificacion de Pell } \\
\text { y Gregory }\end{array}$} & \multicolumn{6}{|c|}{ - EDEMA } & \multirow{2}{*}{\multicolumn{2}{|c|}{ Total }} \\
\hline & & \multicolumn{2}{|c|}{ Leve } & \multicolumn{2}{|c|}{ Moderado } & \multicolumn{2}{|c|}{ Severo } & & \\
\hline & & $\mathbf{n}$ & $\%$ & $\mathbf{n}$ & $\%$ & n & $\%$ & $\mathbf{n}$ & $\%$ \\
\hline \multicolumn{10}{|c|}{ Femenino } \\
\hline \multicolumn{10}{|c|}{ Si Plasma rico en Plaquetas } \\
\hline & A $\|$ & 1 & 6,25 & 0 & 0,00 & 0 & 0,00 & 1 & 6,25 \\
\hline & A IIII & 3 & 18,75 & 0 & 0,00 & 0 & 0,00 & 3 & 18,75 \\
\hline & BI & 2 & 12,50 & 0 & 0,00 & 0 & 0,00 & 2 & 12,50 \\
\hline & $\mathrm{B} \|$ & 4 & 25,00 & 5 & 31,25 & 0 & 0,00 & 9 & 56,25 \\
\hline & B III & 0 & 0,00 & 1 & 6,25 & 0 & 0,00 & 1 & 6,25 \\
\hline & Total & 10 & 62,50 & 6 & 37,50 & 0 & 0,00 & 16 & 100,00 \\
\hline \multicolumn{10}{|c|}{ No Plasma rico en Plaquetas } \\
\hline & A II & 0 & 0,00 & 1 & 6,25 & 0 & 0,00 & 1 & 6,25 \\
\hline & A IIII & 0 & 0,00 & 3 & 18,75 & 0 & 0,00 & 3 & 18,75 \\
\hline & B \| & 0 & 0,00 & 6 & 37,50 & 6 & 37,50 & 12 & 75,00 \\
\hline & Total & 0 & 0,00 & 10 & 62,50 & 6 & 37,50 & 16 & 100,00 \\
\hline \multicolumn{10}{|c|}{ Masculino } \\
\hline \multicolumn{10}{|c|}{ Si Plasma rico en Plaquetas } \\
\hline & A $\|$ & 2 & 20,00 & 1 & 10,00 & 0 & 0,00 & 3 & 30,00 \\
\hline & A IIII & 1 & 10,00 & 0 & 0,00 & 0 & 0,00 & 1 & 10,00 \\
\hline & BI & 2 & 20,00 & 0 & 0,00 & 0 & 0,00 & 2 & 20,00 \\
\hline & $\mathrm{B} \|$ & 2 & 20,00 & 1 & 10,00 & 0 & 0,00 & 3 & 30,00 \\
\hline & B III & 0 & 0,00 & 1 & 10,00 & 0 & 0,00 & 1 & 10,00 \\
\hline & Total & 7 & 70,00 & 3 & 30,00 & 0 & 0,00 & 10 & 100,00 \\
\hline \multicolumn{10}{|c|}{ No Plasma rico en Plaquetas } \\
\hline & A \| & 0 & 0,00 & 1 & 10,00 & 1 & 10,00 & 2 & 20,00 \\
\hline & A IIII & 0 & 0,00 & 2 & 20,00 & 0 & 0,00 & 2 & 20,00 \\
\hline & BI & 0 & 0,00 & 2 & 20,00 & 0 & 0,00 & 2 & 20,00 \\
\hline & B II & 0 & 0,00 & 3 & 30,00 & 1 & 10,00 & 4 & 40,00 \\
\hline & Total & 0 & 0,00 & 8 & 80,00 & 2 & 20,00 & 10 & 100,00 \\
\hline
\end{tabular}

Los resultados obtenidos en relación al dolor no tienen significancia estadística, ya que no se encontró evidencia en el estudio realizado. 
Tabla 7. Relación del dolor POP con el sexo y posición dentaria según Winter.

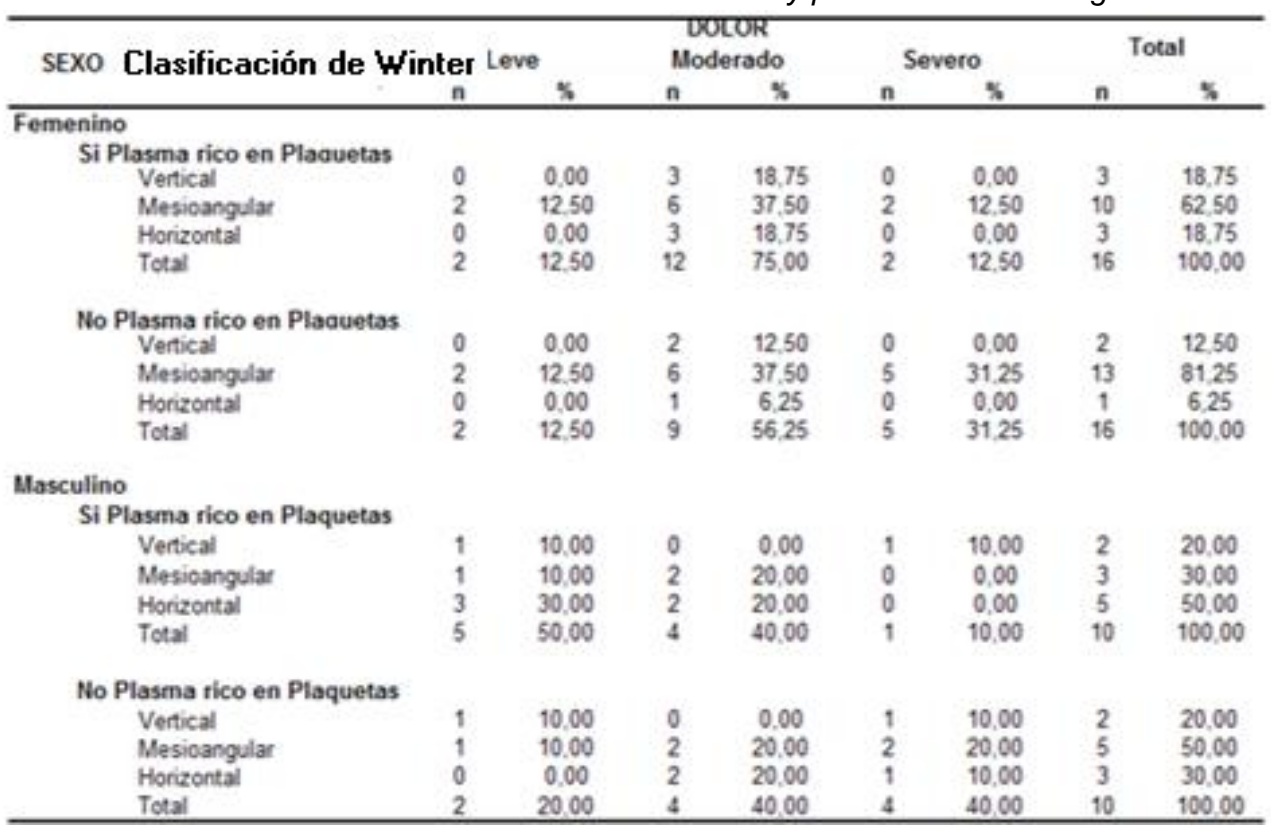

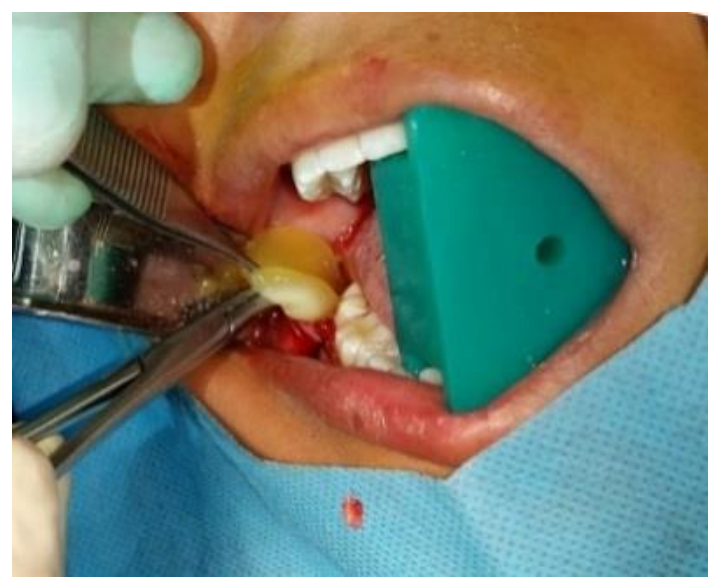

Imagen 1. Colocación intraalveolar de PRPFC en cirugía de tercer molar dentro de la investigación. 
Tabla 8. Relación del dolor POP con el sexo y a la posición dentaria según Pell y Gregory.

\begin{tabular}{|c|c|c|c|c|c|c|c|c|c|}
\hline \multirow[t]{2}{*}{ SEXO } & \multirow{2}{*}{$\begin{array}{l}\text { Clasificación de } \\
\text { Pell y Greqory }\end{array}$} & \multicolumn{2}{|c|}{ Leve } & \multicolumn{2}{|c|}{ Moderado } & \multicolumn{2}{|c|}{ Severo } & \multicolumn{2}{|c|}{ Total } \\
\hline & & $\mathrm{n}$ & 5 & n & $\%$ & $\mathbf{n}$ & $\%$ & $n$ & 5 \\
\hline \multicolumn{10}{|c|}{ Femenino } \\
\hline \multicolumn{10}{|c|}{ Si Plasma rico en Plaquetas } \\
\hline & A II & 0 & 0,00 & 1 & 6.25 & 0 & 0,00 & 1 & 6.25 \\
\hline & A in & 0 & 0,00 & 2 & 12,50 & 1 & 6.25 & 3 & 18,75 \\
\hline & 81 & 1 & 6.25 & 1 & 6.25 & 0 & 0,00 & 2 & 12,50 \\
\hline & B i & 1 & 6.25 & 7 & 43,75 & 1 & 6.25 & 9 & 56,25 \\
\hline & $8=$ & 0 & 0.00 & 1 & 6.25 & 0 & 0.00 & 1 & 6.25 \\
\hline & Total & 2 & 12,50 & 12 & 75,00 & 2 & 12,50 & 16 & 100,00 \\
\hline \multicolumn{10}{|c|}{ No Plasma rico en Plaquetas } \\
\hline & A 1 & 0 & 0,00 & 1 & 6.25 & 0 & 0,00 & 1 & 6,25 \\
\hline & A $\mathbf{n}$ & 0 & 0,00 & 1 & 6.25 & 2 & 12.50 & 3 & 18.75 \\
\hline & 81 & 2 & 12,50 & 7 & 43,75 & 3 & 18,75 & 12 & 75,00 \\
\hline & Total & 2 & 12,50 & 9 & 56.25 & 5 & 31,25 & 16 & 100,00 \\
\hline
\end{tabular}

\section{Masculino}

Si Plasma rico en Plaquetas

A I
A II
Bi
B I
B II
Total

$1 \quad 10,00$

$\begin{array}{lll}10,00 & 1 & 10,00\end{array}$

10,00

0,00

20,00

10,00

50,00

40,00

$\begin{array}{cccc}1 & 10,00 & 3 & 30,00 \\ 0 & 0,00 & 1 & 10,00 \\ 0 & 0,00 & 2 & 20,00 \\ 0 & 0,00 & 3 & 30,00 \\ 0 & 0,00 & 1 & 10,00 \\ 1 & 10,00 & 10 & 100,00\end{array}$

No Plasma rico en Plaquetas

\begin{tabular}{lllllllll} 
A I & 1 & 10,00 & 1 & 10,00 & 0 & 0,00 & 2 & 20,00 \\
A II & 1 & 10,00 & 0 & 0,00 & 1 & 10,00 & 2 & 20,00 \\
BI & 0 & 0,00 & 1 & 10,00 & 1 & 10,00 & 2 & 20,00 \\
B I & 0 & 0,00 & 2 & 20,00 & 2 & 20,00 & 4 & 40,00 \\
Total & 2 & 20,00 & 4 & 40,00 & 4 & 40,00 & 10 & 100,00 \\
\hline
\end{tabular}

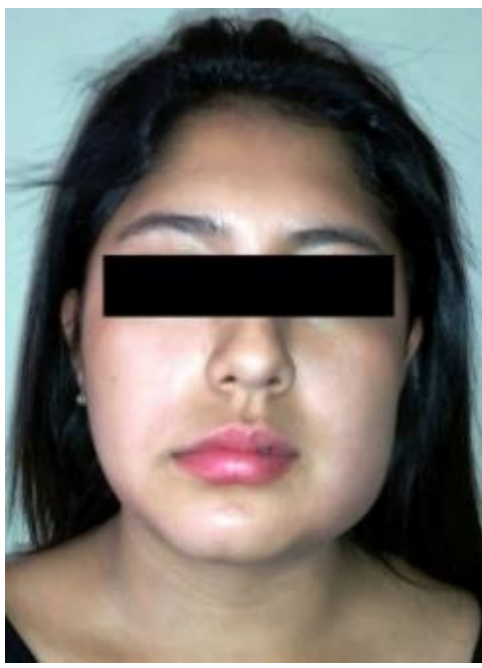

Imagen 2. Muestra el edema al tercer día postoperatorio, notablemente menor en el lado derecho

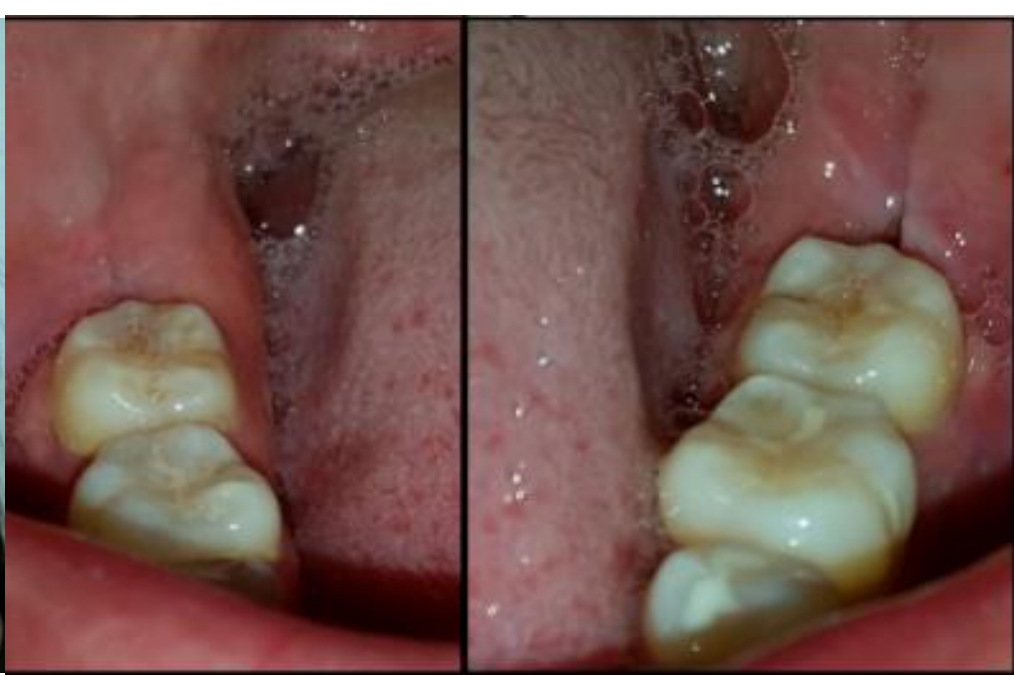

Imagen 3. Muestra la mejor cicatrización del tejido del lado derecho en relación al izquierdo. 


\section{DISCUSIÓN}

Al concluir con esta investigación se obtuvieron los siguientes resultados al aplicar PRPFC en control posoperatorio del tercer molar inferior, presentando el $67,38 \%$ un edema leve y el $34,62 \%$ un moderado. (Imagen 1). En un estudio similar realizado por Víctor Mario Fierro en México en el año 2011, en donde hace un análisis comparativo dentro de la cavidad oral utilizando plasma intraalveolar en el lado derecho únicamente, como resultado la zona en donde se aplicó PRPFC presentó menor edema postoperatorio, menor dolor y mejor cicatrización de los tejidos, lo cual revela similitud en ambos estudios (figura 2).

De igual manera Rami Alissa ${ }^{13}$ en su ensayo clínico randomizado y controlado, llega a conclusiones muy interesantes, que el uso de PRP en cirugía oral, coadyuva a la reparación de los tejidos tanto ósea como de tejido blando, lo que concuerda con este estudio donde también se observó clínicamente una cicatrización avanzada especialmente los tejidos blandos que fueron intervenidos; además Alissa plantea que el PRP actúa en la prevención de osteítis o alveolitis seca, en nuestro estudio se encontró infección secundaria de 3 casos en 3 lados sin PRP

Al hablar acerca de los diferentes métodos y aplicaciones de PRP en base a resultados obtenidos en otras investigaciones dentro de la cirugía oral específicamente en la remoción del terceros molares y en el tratamiento de alveolos post-extracción, James Rutkowski27 en su investigación refiere que los sitios tratados con PRP mejoran durante su primera semana postoperatoria extendiéndose hasta la segunda semana con buenos resultados, Rutkowski ${ }^{27}$ al contrario del autor anterior, afirma que a las seis semanas se podría hacer una evaluación de la densidad ósea, por otra parte el mismo James Rutkowski27 realiza una variación en su proceso investigativo en relación al protocolo de obtención y aplicación del PRP, realiza una centrifugación de 10 minutos y sin realizar la activación plaquetaria exógena con sustancias cálcicas e infiltra directamente en el sitio receptor. En este estudio se aplicó el PRP con activación exógena, colocándolo en forma de gel inestable pero manejable con plaquetas activadas, es decir degranuladas y con factores de crecimiento latentes para actuar en el receptor, esto se consigue al adicionar gluconato de calcio al $10 \%$, que ayuda a un mejor manejo trans-operatorio, asegurando que todo el producto plaquetario obtenido quede incluido en el lugar que deseamos.

El amplio campo de protocolos establecidos para obtención de PRP ha dado paso a muchos otros autores algunos como Eduardo Anitua utiliza el mismo procedimiento de este estudio; pero al replantear o analizar cada uno de estos protocolos, Gernot Weibrich ${ }^{26}$ en su estudio clínico pone a prueba diferentes protocolos de obtención del PRP entre ellos el que se utilizó en esta investigación, Gernot Weibrich ${ }^{26}$ mediante conteos celulares llega a conclusiones muy sólidas y de gran utilidad para quienes aplicamos PRP el afirma que el protocolo de Anitua es el protocolo de mayor validez porque es donde se encuentran mayor cantidad de los factores de crecimiento tipo TGF-B1 es decir el factor beta 1, es el concentrado plaquetario con menor cantidad de células proinflamatorias y con alrededor de 500.000 factores de crecimiento por microlitro en plasma concentrado ${ }^{15}$

\section{CONCLUSIONES}

- Los signos y síntomas postoperatorios del lado que recibió plasma rico en plaquetas con factores de crecimiento fueron más aceptables para el paciente reduciendo el impacto negativo que conlleva, en comparación con el lado que no recibió PRPFC.

- $\quad$ El grado de edema postoperatorio inmediato fue menor al tercer día en los lados que recibieron PRPFC.

- $\quad$ El nivel de dolor en los pacientes operados no tiene diferencia significativa tras ser aplicado PRPFC o haber sido dejado el alveolo vacío sin la colocación de PRPFC.

- La evaluación clínica de la cicatrización de tejidos blandos, demostró que fue más rápida y avanzada tras la aplicación de PRPFC.

\section{REFERENCIAS BIBLIOGRÁFICAS}

1. Vega JA, Días-Esnal B. PRP: Otro punto de vista. Revista Digital del Consell Catalá de l'Esport [Internet]. 2007 [citado 4 ago. 2014]; 42(155):118-22. Disponible en: http://www.apunts.org/es/prp-otro-puntovista/articulo/13112560/ 
2. Beca T, Hernández G, Morante S, Bascones A. Plasma rico en plaquetas: Una revisión bibliográfica. Avances en Periodoncia [Internet]. 2007 [citado 8 ago. 2015]; 19(1): 39-52. Disponible en: http://scielo.isciii.es/scielo.php?script=sci arttext\&pid=S1699-65852007000200005\&lng=es.

3. Reyes M, Montero S, Cifuentes J, Zarzar Emilio. Actualización de la técnica de Obtención y Uso del Plasma Rico en Factores de Crecimiento (P.R.G.F). Revista Dental de Chile [Internet]. 2002 [citado 28 ago. 2014]; 93(2): 25-28. Disponible en:

\section{http://www.revistadentaldechile.cl/temas\%20agosto\%202002/PDFs agosto 2002/Actualizacion\%20de\%20la} \%20Tecnica\%20de\%20Obtencion...\%20.pdf

4. Montón Echeverría J, Pérez Redondo S, Gómez Bajo GJ. Experiencia 14andomi en el empleo de factores de crecimiento autólogos obtenidos de plasma rico en plaquetas. Cirugia Plastica Ibero-Latinoamericana [Internet]. 2007 [citado 5 septiembre 2014]; 33(3): 155-162. Disponible en: http://scielo.isciii.es/scielo.php?script=sci arttext\&pid=S0376-78922007000300002\&lng=es.

5. Fernández RG, López M, Ruíz E. Plasma rico en factores de crecimiento en cirugía bucal. Presentación de caso clínico. Revista Odontológica Mexicana [Internet]. 2005 [citado 25 ago 2014]; 9(3): 141-146. Disponible en: http://www.medigraphic.com/pdfs/odon/uo-2005/uo053f.pdf

6. Fierro-Serna VM, Martínez R, Hídalgo J, Toranzo J, Pozos A. Colocación de plasma rico en factores de crecimiento postextracción de terceros molares inferiores: Reporte de un Caso. Revista Odontológica Mexicana [Internet]. 2011 [citado 8 sep 2014]; 15(2): 109-114. Disponible en: http://www.medigraphic.com/pdfs/odon/uo2011/uo112f.pdf

7. Nikolidakis D, Janse J. The Biology of Platelet-Rich Plasma and Its Aplication in Oral Surgery: Literature Review. TISSUE ENGINEERING [Internet]. 2011 [citado 9 sep 2014]; 14(3): 248-258. Disponible en: http://online.liebertpub.com/doi/pdf/10.1089/ten.teb.2008.0062

8. Albanese A, Licata M, Polizzi B, Campisi G. Platelet-Rich Plasma in dental and oral surgery: From the wound healing to bone regeneration. Immunity \& Ageing [Internet]. 2013 [citado 12 sep 2014]; 10-23. Disponible en: http://www.ncbi.nlm.nih.gov/pmc/articles/PMC3683340/

9. Del Fabbro M, Bortolin M, Taschieri S. Is autologous platelet concentrate beneficial for post-extraction socket healing? A systematic review. Int J Oral Maxillofac Surg [Internet]. 2011 [citado 15 sep 2014]; 40(9): 890-891Disponible en: http://www.ncbi.nlm.nih.gov/pubmed/2160208

10. González M, Arteaga-Vizcaíno M, Benito M, Benito M. Aplicación del plasma rico en plaquetas (PRP) y sus derivados en implantología dental y cirugía plástica. Invest. Clín [Internet]. 2012 [citado 18 sep 2014]; 53(4): 408-418. Disponible en: http://www.scielo.org.ve/scielo.php?script=sci arttext\&pid=S053551332012000400009\&lng=es.

11. González S, Ortíz G. Plasma Rico en Plaquetas: Una alternativa para acelerar el proceso de cicatrización Ósea. Revista CES Odontología [Internet]. 2004; 2012 [citado 20 sep 2014]; 17(1):71-74. Disponible en: http://revistas.ces.edu.co/index.php/odontologia/article/viewFile/472/268

12. Rodríguez J, Palomar M, García J. Plasma rico en plaquetas: fundamentos biológicos y aplicaciones en cirugía maxilofacial y estética facial. Revista Española de Cirugía Oral y Maxilofacial [Internet]. 2012 [citado 22 sep 2014]; 34(1):8-17. Disponible en: http://scielo.isciii.es/pdf/maxi/v34n1/original2.pdf

13. Alissa R, Esposito M, Horner K, Oliver R. The Influence of Platelet-Rich Plasma on the healing of extraction sockets: An explorative Randomized clinical trial. Eur J Oral Implantol [Internet]. 2010 [citado 23 sep 2014]; 3(2): 121-134 Disponible en: http://www.ncbi.nlm.nih.gov/pubmed/20623037

14. Martínez JM, Cano J, Gonzalo JC, Campo J, Esparza G, Seoane JM. ¿Existen riesgos al utilizar los concentrados de Plasma Rico en Plaquetas (prp) de uso ambulatorio?. Medicina Oral [Internet]. 2002 [citado 25 sep 2014]; 7: 375-390. Disponible en: http://www.medicinaoral.com/pubmed/medoralv7 i5 p375.pdf

15. XVI Jornadas de Actualización en Cirugía Ortopédica y Traumatología. Plasma rico en plaquetas [Internet], Murcia-España; 2012 [actualizado 13 mar 2013; citado 27 sep 2014]; Disponible en: www.youtube.com/watch?V=Q9VyJszXosA

16. Lee UL, Jeon SH, Park JY, Choung PH. Effect of Platelets Rich Plasma on Dental Stem Cells Derived From Human Impacted Third Molars. Regen Med [Internet]. 2011 [citado 6 sep 2014];6(1):67-79. Disponible en: http://www.ncbi.nlm.nih.gov/pubmed/21175288

17. Lindeboom J, Mathura K, Aartmanl, Kroon F, Milstein D, Ince C. Influence of The Application of PlateletEnriched Plasma in Oral Mucosal Wound Healing. Clinical Oral Implantology [Internet]. 2006 [citado 8 sep 2014]; 18(1): 133-39. Disponible en: http://onlinelibrary.wiley.com/doi/10.1111/j.16000501.2006.01288.x/abstract

18. Mendieta TR, Alvarado JC, Negrete J. Utilidad del Plasma Rico en Plaquetas y Factores de Crecimiento en Defectos Óseos, experiencia en el Hospital Regional Lic. Adolfo López Mateos del ISSSTE. Acta Ortopédica 
Mexicana [Internet]. 2007 [citado 12 sep 2014]; 21(5): 256-260. Disponible en: http://www.medigraphic.com/pdfs/ortope/or-2007/or075f.pdf

19. Jankovic SM, Zoran AM, Vojislav LM, Bozidar DS, Kenney BE. The Use Of Platelet-Rich Plasma in Combination with Connective Tissue Grafts Following Treatment of Gingival Recessions. Periodontology $\begin{array}{llllllll}\text { [Internet]. } 2007 \quad \text { [citado } 15 & \text { sep 2014]; } & 4(1): & 63-71 . & \text { Disponible en: }\end{array}$ http://perio.quintessenz.de/perio 200701 s0063.pdf

20. Santana RB, Santana CM. A Clinical Comparission of Guided Bone Regeneration with Platelet-Derived Growth Factor Enhanced Bone Ceramic Versus Autogenus Bone Block Grafting. The International Journal of Oral \& Maxillofacial Implant [Internet]. 2015 [citado 10 ene 2015]; 30(3). 700-06. Disponible en: http://www.ncbi.nlm.nih.gov/pubmed/26009922

21. Escobar T, Almeida e Sousa J, Portela A, Vasconcels M, Faria de Almeida R. The Effect of A Biphasic Calcium Phosphate on Bone Healing_A Pilot Study in Rats. Department of Oral Surgery And Medicine, School of Dental Medicine University Oporto. 2014; 29(3):1322-31

22. Guo Han Lin. Interdisciplinary Approach for Treating a Large Through and Through Periapical Defect Using Guided Tissue Regeneration: A Case Report. The International Journal of Periodontics \& Restorative Dentistry $\begin{array}{lllllll}\text { [Internet]. } 2014 \quad \text { [citado } 10 \text { ago 2014]; } & 34(1): e 1-e 8 . & \text { Disponible en: }\end{array}$ http://www.quintpub.com/journals/prd/abstract.php?article id=13804\#.VetY9xF Okp

23. Kiany F, Moloudi F. Amnion Membrane As A Novel Barrier In Th Treatment Of Intrabony Defects: A Controlled Clinical Trial. The International Journal Of Oral \& Maxillofacial Implants [Internet]. 2015 [citado 8 oct 2014]; 30(3): 639-47. Disponible en:

http://www.unboundmedicine.com/medline/citation/26009915/Amnion membrane as a novel barrier in the treatment of intrabony defects: a controlled clinical trial

24. Marx R. rhbmp-2/ACS Grafts Versus Autogenous Cancellous Marrow Grafts In Large Vertical Defects Of The Maxilla: An Unsponsored Randomized Open-Label Clinical Trial. Oral \& Craniofacial Tissue Engineering. $2011 ; 1(1)$. P. 33-41

25. Koerdt S. Expression Of Growth Factors During The Healing Process Of Alveolar Ridge Augmenting Procedures Using Autonegus Bone Grafts In Combination With GTR And An Anorganic Bovine Bobe Substitute. Clinical Oral Investigation. 2014; 18: p. 179-88.

26. Weibrich Gernot. Comparision of Point of Care Methods for Preparation of Platelet Concentrate. The International Journal Of Oral \& Maxillofacial Implants. 2012; 27(4): P. 762-769. Disponible En: Ebsco Host

27. Rutkowski JL, Johnson DA, Radio NM, Fennell JW. Platelet Rich Plasma To Facilitate Wound Healing Following Tooth Extraction. Journal Of Implantology [Internet]. 2010 [citado 26 sep 2014]; 36(1):11-23. Disponible En: http://www.joionline.org/doi/pdf/10.1563/AAID-JOI-09-00063. 